\title{
EXPERIENCES WITH LIGHT WEIGHT FIXED WING AERIAL MAPPING UAVs
}

\author{
W. Mayr ${ }^{a}$ \\ a GerMAP GmbH, Justinus-Kerner-Str. 8, 73642 Welzheim, Germany - werner.mayr@germap.com
}

Commission I, ICWG I/5b

KEY WORDS: UAV/UAS/RPAS, aerial mapping, sensor platform

\begin{abstract}
:
UAVs seem to be the next "cloud" like topic, not only in geomatics. Unmanned Airborne Vehicles are not a wonder-tool, but a complementary approach to resolve some tasks more efficiently than before or at all. Since 2006 we commercially apply fixed wing, light weight UAVs for aerial mapping purposes. In this paper we like to share our experiences with UAVs of less than $5 \mathrm{~kg}$ and illuminate some limitations as well as potentials. Whereas multicopters seem to be in use everywhere, fixed wing UAVs more frequently seem to be applied in specific and geospatially oriented applications. Having processed several hundred UAV aerial mapping projects there forms a stable picture of this technology. Our impressions on durability, handling, and reliability of fixed wing UAVs get presented. We report on our day-to-day experiences and point to often simple hurdles to overcome. Various cameras were flown, different approaches of handling their geometries with different software packages were undertaken. Remarks to achieved geometric accuracies as well as the consequences of using dual frequency GPS instead of simple yet great single frequency GPS are discussed. All of this is packed into the subsequent paper.
\end{abstract}

\section{INTRODUCTION}

A number of European companies apply and focus on services based on unmanned survey aircrafts. There is also a number of manufacturers of unmanned aircraft systems (UAS). And yet quite many publications report on use and application examples of UAS, which are also called RPAS (= remotely piloted aircraft system), e.g. (Eisenbeiss, 2011), (Grenzdörffer, 2011), (Mayr, 2009, 2011, 2013), (Colomina, 2014). So, three major representatives of society, service providers, system providers, and academia tend to become deeply involved into activities based on and related to the use of some unmanned, flying vehicle. In our geospatial domain we are interested in applicability of RPAS for delivering remote sensing data, often aerial imagery.

The two major types of aircrafts for RPAS are rotary-wingbased aircrafts, so-called multicopters, and fixed wing aircrafts or simply airplanes. This paper reports on use of fixed wing aircrafts. Another categorization of the flying vehicle uses its "maximum take-off weight" (MTOW) and impacts not only its flight dynamics but also administrative aspects, such as insurance and legal matters.

It seems, RPAS are "everywhere you look", as one could see on the recent exhibition Intergeo 2014, Oct.7-9, 2014, Berlin, Germany. All major players in the geomatics industry offer some sort of RPAS-response to the community.

This paper presents some experiences and views of the author who commercially operates light weight aerial mapping fixed wing RPAS since 2006. Aspects of flying as well as data processing shall show how it works.

\section{RPAS - REMOTELY PILOTED AIRCRAFT SYSTEM}

A RPAS in this context consists of an unmanned airborne vehicle (UAV) that a human pilot may control at any time and that flies automatically when on mission, all of which within the visible line of sight (VLOS) from pilot to UAV. As the acronym RPAS expresses, the pilot is responsible ultimately. It thus is a consequence to enable the pilot taking over full flight control at any time s/he likes or needs to do so. When the UAV is on e.g. an aerial imaging mission the autopilot steers the UAV on its pre-defined path and returns the UAV to its home, meaning starting, position while its actual versus planned flightpath is being visualized in real-time on the RPAS-groundstation screen, which usually another person observes while the pilot keeps her/his eyes on the UAV at all the times.

Air traffic law is national law and follows some internationally agreed upon rule sets. For more detail on this see (Colomina, 2014). In Europe, RPAS are often sorted into weight classes, when it comes to flight permits. In Scandinavia for example there are such classes as la for up to $2.5 \mathrm{~kg}$ MTOW and $\mathrm{lb}$ for up to $7 \mathrm{~kg}$, while in Germany there are classes of up to $5 \mathrm{~kg}$, up to $25 \mathrm{~kg}$, and up to $150 \mathrm{~kg}$.

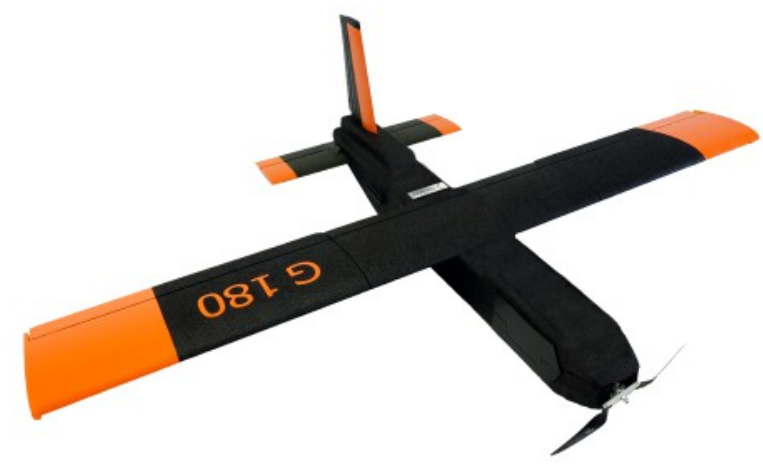

Figure 1. G180 RPAS, $3.7 \mathrm{~kg}$ MTOW, $1.3 \mathrm{~km}$ VLOS range 
However, most all commercially operated RPAS belong to the lowest weight classes, as this gives the easiest access to obtain a flight permit which still bears several operational constraints.

Figure 1 shows as an example of an light weight RPAS the G180 manufactured by GerMAP, Germany, with less than $5 \mathrm{~kg}$ MTOW, $180 \mathrm{~cm}$ wingspan, and a VLOS range of approximately $1.3 \mathrm{~km}$. One can replace its wings to a wingspan of $220 \mathrm{~cm}$, a G220, and even extend its VLOS range somewhat. G180 resp. G220 can cover an area of e.g. $1.5 \mathrm{~km}^{2}$ flown in approx. $30 \mathrm{~min}$ when operating in $100 \mathrm{~m}$ above ground level (AGL) with $80 \%$ along-track and $70 \%$ across-track overlaps, equipped with one nadir looking 16.2 Mpix RicohGR camera having 4828 pixels across-track and a focal length of $18.3 \mathrm{~mm}$ delivering from this AGL close to $3 \mathrm{~cm}$ ground sampling distance (GSD).

Figure 2 displays an even lighter RPAS, the SmartOneC with $1.2 \mathrm{~kg}$ MTOW manufactured by SmartPlanes, Sweden, detachable wing halves and $120 \mathrm{~cm}$ wingspan. It thus fits into the 1a class of flight permit for Scandinavia.

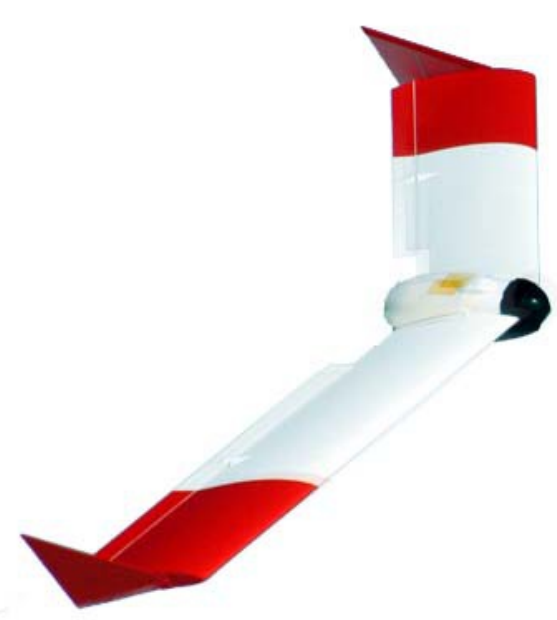

Figure 2. SmartOneC (S1C), $1.2 \mathrm{~kg}$ MTOW, 800m VLOS range

\section{RPAS - EXPERIENCES AND CONSIDERATIONS}

We fly with fun but not for fun, since the goal is always to generate aerial images for photogrammetric data processing. What we are after, are deliverables such as orthomosaics, digital surface or terrain models, and derivatives thereof such as volumes, profiles, or contours. Available professional photogrammetric software such as Trimble-Inpho's MATCH suite of software is capable of processing RPAS-imagery. It might be required to know some details of the software applied in order to correctly set proper parameters.

At time of starting in 2006 with RPAS-Mapping or UAVMapping, as one may call it, there was no RTK equipment on a component level available for integration into small, light weight UAVs. Thus, many commercial RPAS operate based on single phase GPS, i.e. without RTK, still. Yet, one can produce deliverables in very good quality, see Figure 3. Depending on the geometry of the block or blocks to be flown, one distributes a number of ground control points (GCP) and measures them via differential GPS. Also, with RTK one will want to perform some sort of quality check and control, and this requires a few GCPs anyway. Measuring a few more GCPs which due to the small size of area covered by RPAS-Mapping, as compared to manned aircraft aerial survey, often is correlated to short dis- tances between GCPs and thus easily possible to be accomplished. RTK certainly will bring even more performance to RPAS-Mapping, but as of now it seems to increase the entry price level into this technology above the threshold for many users.

From quite many and different projects we obtained a stable confirmation in achieving a planimetric accuracy of approx. 0.5 GSD and an accuracy in height of approx. 0.8 to 1.5 GSD if image data gets properly processed. This is valid for GSDs as small as $4 \mathrm{~cm}$ or larger. GSDs of smaller size than $4 \mathrm{~cm}$ have the planimetric accuracy sort of asymptotically approaching 1 GSD and height accuracy is drifting towards 1.5 GSD. For data processing we apply the aforementioned MATCH suite of software including OrthoMaster, OrthoVista, and DTMaster, but as as well UASMaster or PhotoScan Pro from Russian AgiSoft. Table 1 shows of some typical projects their main parameters and includes planimetric and height accuracies obtained.

\begin{tabular}{|l|c|c|c|c|c|c|c|}
\hline $\begin{array}{l}\text { Appli- } \\
\text { cation }\end{array}$ & $\begin{array}{c}\text { B1 } \\
\text { oc } \\
\text { ks }\end{array}$ & $\begin{array}{c}\text { Aerial } \\
\text { images }\end{array}$ & $\begin{array}{c}\text { GSD } \\
{[\mathrm{cm}]}\end{array}$ & $\begin{array}{c}\text { DSM- } \\
\text { spacin } \\
\mathrm{g}[\mathrm{cm}]\end{array}$ & $\begin{array}{c}\text { Plani- } \\
\text { metric } \\
\text { acc. } \\
\pm[\mathrm{cm}]\end{array}$ & $\begin{array}{c}\text { Height } \\
\text { acc. } \\
\pm[\mathrm{cm}]\end{array}$ & $\begin{array}{c}\text { Area } \\
{[\mathrm{ha}]}\end{array}$ \\
\hline Landfill & 1 & 193 & 7.5 & 50 & 3 & 3.1 & 33 \\
\hline Landfill & 3 & 691 & 8 & 35 & 3.5 & 5.2 & 113 \\
\hline Landfill & 3 & 983 & 6 & 50 & 2.5 & 3.8 & 117 \\
\hline Golf & 4 & 1126 & 8 & 50 & 3 & 3.8 & 170 \\
\hline Golf & 2 & 332 & 7.5 & 50 & 3 & 7.2 & 70 \\
\hline Golf & 2 & 346 & 7.5 & 50 & 3.5 & 6.4 & 88 \\
\hline Quarry & 3 & 707 & 10 & 30 & 2.5 & 6.0 & 117 \\
\hline Cadastre & 2 & 445 & 6 & 40 & 2 & 1.5 & 64 \\
\hline New home & 3 & 557 & 6 & 40 & 3 & 6.8 & 104 \\
\hline
\end{tabular}

Table 1. Typical projects and their main parameters

Facing the type of cameras used in RPAS vs. the many orders of magnitudes more expensive and highly sophisticated large format digital optical sensors such as ADS, DMC, or UltraCam these simple cameras deliver surprisingly good imagery, very much usable in photogrammetric measurement techniques.

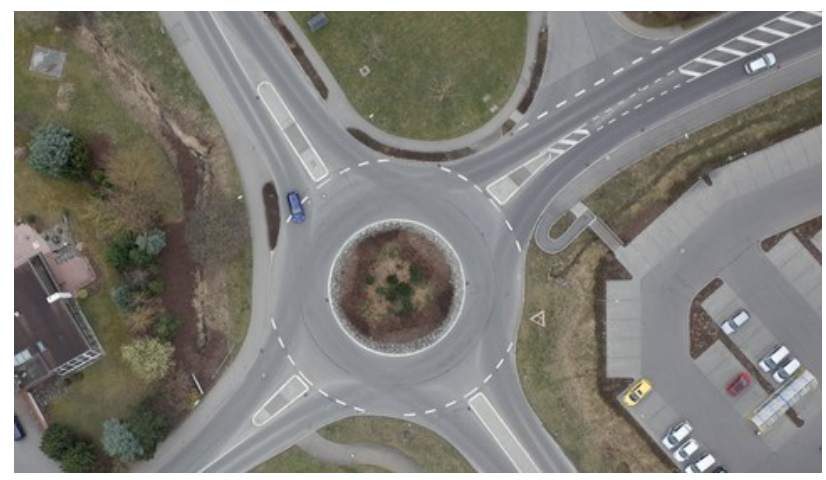

Figure 3. Orthomosaic, $3 \mathrm{~cm}$ GSD, RicohGR, flown with S1C

A little survey on change of focal length of a Canon S100 camera over time is shown in Figure 4. This camera gets next to other cameras used e.g. in S1C, G180, or GerMAP's G212 but 
has no fixed focus and thus has to be firmware-driven put into infinite auto-focus lock position each time it gets applied in a RPAS-Mapping mission.

From February to July 2013 we selected 18 blocks flown with the identical Canon S100. Each time the Canon S100 gets used, its firmware moves the lens to its - as the firmware thinks identical numerical position representing infinity. The actual focal length, however, varied between $5.3696 \mathrm{~mm}$, see number 4 in Fig. 4, and $5.3881 \mathrm{~mm}$, see number 5 in Fig. 4. For such a small and simple camera, this variation could be considered as pretty small. Yet, one needs to model it in the photogrammetric data processing path.

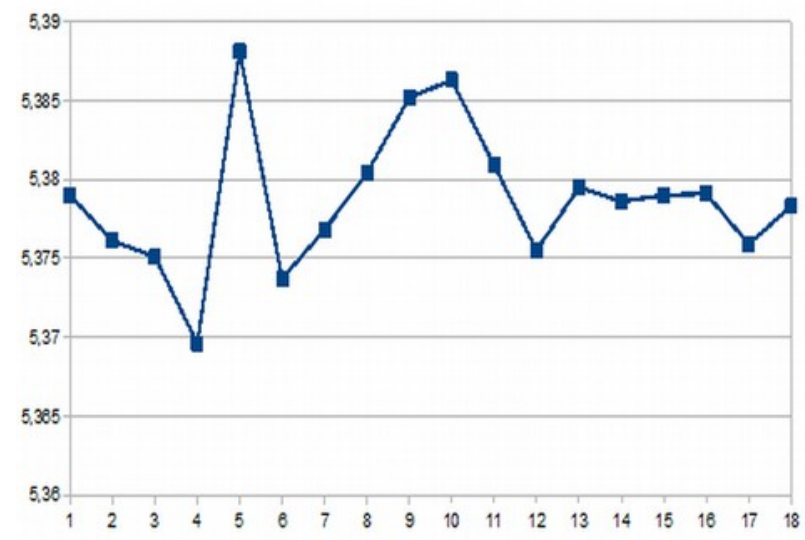

Figure 4. Focal length time series of the same Canon S100

One way to cope with this variation is to get mentally away from a static metric camera model towards a dynamic nonmetric camera model with varying focal lengths and distortions as per switch-on-off cycle, thus having the need to apply selfcalibration during aerial triangulation for on-line determination of the frequently changing interior orientation parameters. However, this requires some measurable GCPs.

Table 2 compares the two mentioned cameras Canon S100 and RicohGR, see Figure 5. For flight planning the two values focal length vs. pixel pitch and pixels across track are most important ones. Here, the younger RicohGR is an advantage as it delivers from approximately $25 \%$ higher AGL the same GSD, which in turn reduces the number of photo strips to fly, i.e. RicohGR delivers an increase in efficiency. Moreover, the larger pixelarea for photon collection in RicohGR improves image quality to some extent.

\begin{tabular}{|l|c|c|}
\hline Item & Canon S100 & RicohGR \\
\hline Type of focal length & Variable & Fixed \\
\hline Focal length (FL) [mm] & 5.3696 to 5.3881 & 18.3 \\
\hline Pixel pitch (PP) [micron] & 1.82 & 4.8 \\
\hline Ratio FL / PP & 2956 & 3812 \\
\hline Chip type & CMOS & CMOS \\
\hline Chip rows = along track & 3000 & 3264 \\
Chip columns = across track & 4000 & 4828 \\
\hline Weight [gram] & 189 & 243 \\
\hline
\end{tabular}

Table 2. Canon S100 vs. RicohGR
Figure 2 depicts the Canon S100 and RicohGR cameras used in RPAS operations. Of course, other brands get applied as well and might have e.g. larger Mpixel values, but also higher weights to carry in the UAV. The across track coverage and the ratio FL / PP are the parameters which count when optimizing the flightpath, and the weight of the camera directly influences flight duration; the more MTOW weight the less flying time.
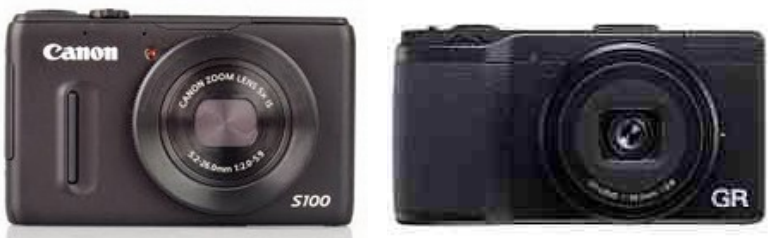

Figure 5. Canon S100 (left) and RicohGR (right)

Yet another consideration is with respect to permitted AGL. In Europe, the standard AGL may vary between $100 \mathrm{~m}$ to $150 \mathrm{~m}$. Special, singular permits are required for higher AGL. Thus, for standard RPAS-flight permits valid for flight missions up to 150 $\mathrm{m}$ AGL it is worth an evaluation if a high performance UAVcamera such as iXU 150 from PhaseOne with 8280 × 6208 pixels, 5.3 micron pixel pitch, and 750 grams for the camera housing only plus one of several lenses of different, but fixed focal lengths between $28 \mathrm{~mm}$ and $280 \mathrm{~mm}$ having lens-weights between 428 grams to 1600 grams is the appropriate tool.

To further optimize flight performance one can think of reducing the amount of time necessary to fly for an area. Due to the light weight of the UAV wind may bounce the flying vehicle sometimes quite a bit. Of course, this happens during exposure. Even with exposures of $1 / 2000 \mathrm{sec}$ and F2.8 one will get, sometimes, blurred imagery. This may be used for aerial triangulation, however not for orthoprojection. One solution to this is a stabilized mount. The expectation is that such a stabilization keeps the camera nadir looking even if wind causes bigger banking angles for the airplane. At GerMAP we developed a 2axis stabilization capable of carrying 2 Canon S100, see Figure 6.

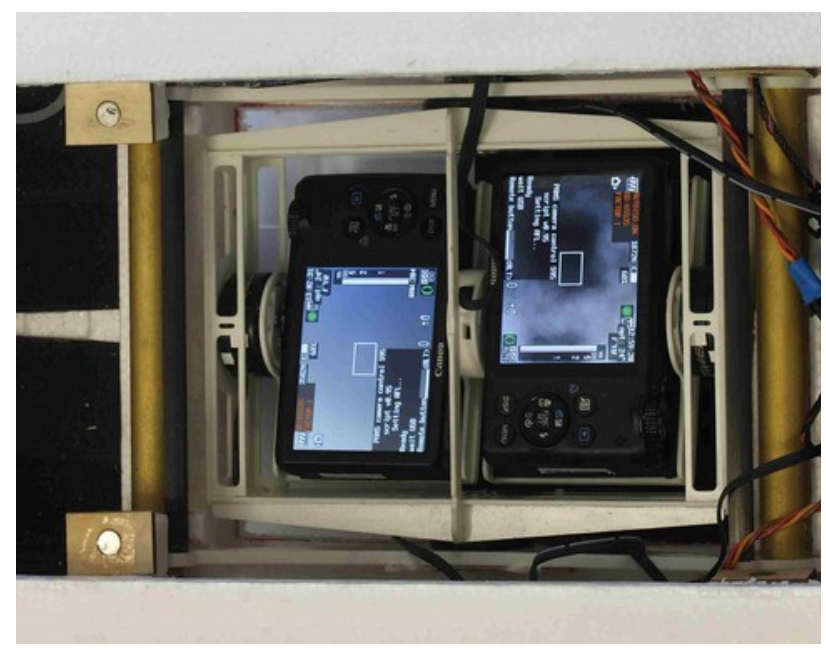

Figure 6. 2-axis stabilization mount for 2 Canon S100

One can pre-set the camera plane to horizontal or into oblique position. This way one can either double the swath width or e.g. 
operate a RGB Canon S100 and a NIR modified Canon S100 in parallel nadir looking mode. Figure 6 shows such a 2 -axis stabilization mount. Together with 2 Canon S100 it has a weight of 835 grams and stabilizes in roll and pitch. Such a mount greatly reduces the need for very high across track overlap down to some $40 \%$ only. This minimizes required time per area and increases number of areas flown per day. In double swath wide mode having the two cameras slightly oblique but overlapping looking one can even further reduce the flying time needed to cover an area.

Since this dual-camera-mount is approx. $23 \mathrm{~cm}$ wide and $26 \mathrm{~cm}$ long it does not fit into e.g. a G180. We had to find another airplane with a bigger payload bay and decided to go with a big flying wing. GerMAP's G212 is the result, see Figure 7. It has $212 \mathrm{~cm}$ wingspan and a MTOW of $3.8 \mathrm{~kg}$ of which $1.2 \mathrm{~kg}$ may be payload.

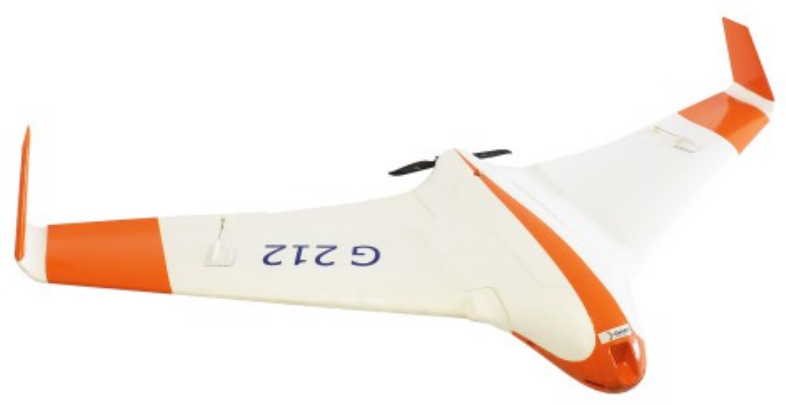

Figure 7. G212, MTOW $3.8 \mathrm{~kg}$, remote sensing sensor platform, can alternatively carry different camera configurations

Both, G212 and G18/G220, can fly for 45 min which is more than enough to cover photogrammetrically all areas possible to be flown with in VLOS range.

\section{CONCLUSIONS}

RPAS for aerial mapping and remote sensing is applicable and delivers reliably accountable deliverables. It complements in many areas existing but latent market opportunities. Common photogrammetric flight mimic is implemented and related data processing in place. High performance professional photogrammetric software as well as newly appearing packages such as PhotoScan Pro from Agisoft or Pix4D deliver good results.

The applications are manifold, and they dictate the appropriate RPAS-tool to solve different engineering tasks. Thus fixed wing as well as multi-rotor wing UAVs will co-exist and have their benefits for specific types of tasks.

The technology of unmanned aerial vehicles suffers to some extent in reputation due to a non-commercial use, but also abuse by some individuals. Commercial use of RPAS has the potential to build up and strengthen an excellent reputation of UAV-technology when applied with ethics and to the benefit of the society.

National legislations and international harmonization of use of RPAS will have a major impact on commercial growth of RPAS-technology. Policies for RPAS operations are treated on an European level, e.g. (Kämpfe, 2013).
Demands from market places, e.g. agriculture, forestry, civil engineering, insurances, civil protection, geological prospection, and others, might make use of RPAS as a "simple tool" for data collection. Common to all is the need to georeference acquired data and ascertain quality. This is the domain of geomatics-engineers.

Integration of yet more versatile sensors, miniaturization, and simplification of user-interfaces will further foster the applicability of RPAS.

May all RPAS pilots always have safe and happy landings!

\section{REFERENCES}

Colomina, I. \& Molina, P., 2014, Unmanned aerial systems for photogrammetry and remote sensing: A review. ISPRS Journal of Photogrammetry and Remote Sensing 92 (2014) Elsevier, pp. 79-97.

Eisenbeiss, H., 2011: The Potential of Unmanned Aerial Vehicles for Mapping. Proceedings Photogrammetric Week 2011. Wichmann Verlag, pp.147-154.

Grenzdörffer, G. \& Niemeyer F., 2011: UAV based BRDFMeasurements of Agricultural Surfaces with Pfiffikus. Proceedings of the International Conference on Unmanned Aerial Vehicle in Geomatics (UAV-g), Zurich, Switzerland, September 14-16, 2011, ISSN 1682-1777

Kämpfe, F., 2013: RPAS in international, European and national air law - a short overview. RPAS/UAS Workshop - A challenge for international, European and national air law; presentation; Cologne May23/24, 2013

Mayr, W., 2009: PAMS - Personal Aerial Mapping System. White Paper, download from www.germap.com $\backslash$ downloads

Mayr, W., 2011: Unmanned Aerial Systems in Use for Mapping at Blom. Proceedings Photogrammetric Week 2011, Wichmann Verlag, pp.125-134.

Mayr, W., 2013: Unmanned Aerial Systems - for the Rest of Us. Proceedings Photogrammetric Week 2013, Wichmann Verlag, pp.151-163.

www:

http://industrial.phaseone.com/iXU camera system.aspx http://www.germap.com http://www.sensefly.com http://www.smartplanes.se http://www.trimble.com http://www.uvs-info.org for RPAS Glossary 120812 